\title{
The Magnetic Field Effects on Spin Polarization of T-Shaped Double Quantum Dots Coupled to Ferromagnetic Leads
}

\author{
K.P. WÓJCIK* AND I. WEYMANN \\ Faculty of Physics, Adam Mickiewicz University, Umultowska 85, 61-614 Poznań, Poland
}

\begin{abstract}
We analyze the spin-dependent conductance and spin polarization of a double quantum dot in a T-shape configuration coupled to ferromagnetic leads in the presence of external magnetic field. The calculations are performed with the aid of the numerical renormalization group method. We show that in the antiparallel configuration, finite magnetic field can give rise to the full spin polarization of the current, which can be controlled by tuning the dots' levels. On the other hand, for parallel configuration enhanced spin polarization can be generated by an exchange field due to the presence of ferromagnetic leads and can be also tuned by changing level position or external magnetic field. The magnetic field can be thus used to improve the spin-resolved properties of the system.
\end{abstract}

DOI: $10.12693 /$ APhysPolA.127.222

PACS: 73.23.Hk, 73.63.Kv, 85.75.-d

\section{Introduction}

Transport properties of double quantum dots (DQDs) have recently attracted a lot of attention [1]. Since the behavior of such systems mimics the behavior of real molecules, DQDs provide ideal playground to examine various correlations at the nanoscale. When only one of the dots is directly coupled to the leads, while the second dot is coupled indirectly through the first dot, the interference between different conduction paths can give rise to the Fano effect [2]. On the other hand, in the case of strong coupling between the DQD and the leads, the electronic correlations can give rise to the Kondo effect [3]. In fact, transport properties of T-shaped DQDs are determined by the interplay of the Fano and Kondo effects $[4,5]$. Another interesting effect occurs in the presence of external magnetic field. It was shown that the conductance through the system can be then fully spin polarized, and the sign and degree of spin polarization can be controlled by the position of the DQD's levels [6].

In this paper we study the spin-polarized conductance of T-shaped DQDs coupled to ferromagnetic leads in the presence of external magnetic field. It is known that the presence of ferromagnetic leads gives rise to an exchange field that acts in a similar way to an external magnetic field, splitting the dots' levels $[7,8]$. By using the numerical renormalization group (NRG) method $[9,10]$, we show that one can obtain full spin polarization $\mathcal{P}$ of the linear conductance in DQDs with ferromagnetic contacts without the need to apply magnetic field. We also analyze how the presence of external magnetic field modifies the spin-resolved transport properties of the system.

${ }^{*}$ corresponding author; e-mail: kpwojcik@amu.edu.pl

\section{Model and method}

We consider DQD in a T-shape configuration coupled to ferromagnetic leads, whose magnetizations can form either parallel or antiparallel configuration, see Fig. 1. The Hamiltonian of the system has the form, $H=H_{\mathrm{F}}+H_{\mathrm{T}}+H_{\mathrm{DQD}} \cdot H_{\mathrm{F}}=\sum_{r=L, R} \sum_{\boldsymbol{k} \sigma} \varepsilon_{r \boldsymbol{k} \sigma} c_{r \boldsymbol{k} \sigma}^{\dagger} c_{r \boldsymbol{k} \sigma}$ is the Hamiltonian of ferromagnetic leads, $H_{\mathrm{T}}=$ $\sum_{r=L, R} \sum_{\boldsymbol{k} \sigma} V_{r \boldsymbol{k} \sigma}\left(d_{1 \sigma}^{\dagger} c_{r \boldsymbol{k} \sigma}+c_{r \boldsymbol{k} \sigma}^{\dagger} d_{1 \sigma}\right)$ is the tunneling Hamiltonian and the DQD Hamiltonian reads

$H_{\mathrm{DQD}}=\sum_{j \sigma} \varepsilon_{j \sigma} n_{j \sigma}+\sum_{\sigma} t\left(d_{1 \sigma}^{\dagger} d_{2 \sigma}+\right.$ H.c. $)+U_{2} n_{2 \uparrow} n_{2 \downarrow}$,

with $n_{j \sigma}=d_{j \sigma}^{\dagger} d_{j \sigma}$. Here, $c_{r \boldsymbol{k} \sigma}\left(d_{j \sigma}\right)$ annihilates an electron with spin $\sigma$, carrying momentum $\hbar k$ in lead $r$ (located on dot $j$ ), correspondingly, and $\varepsilon_{r \boldsymbol{k} \sigma}\left(\varepsilon_{j \sigma}\right)$ is the respective single-particle energy. We assume that only the second dot is interacting, with $U_{2}$ denoting the Coulomb correlation parameter. In the presence of magnetic field, the dots' levels become split, $\varepsilon_{j \sigma}=\varepsilon_{j}+\sigma B / 2$, where $B$ is the external magnetic field applied along the $z$-th direction and $g \mu_{\mathrm{B}} \equiv 1$. The coupling between the first dot and the lead $r$ is given by $\Gamma_{r \sigma}=\sum_{\boldsymbol{k}} \pi \rho_{r \sigma}\left|V_{r \boldsymbol{k} \sigma}\right|^{2}$, where $\rho_{r \sigma}$ denotes the respective spin-resolved density of states. The coupling can be rewritten as $\Gamma_{r \sigma}=(1+\sigma p) \Gamma_{r} / 2$, where $p$ is the spin-polarization of ferromagnets $\left(p_{\mathrm{L}}=\right.$ $\left.p_{\mathrm{R}} \equiv p\right)$, and $\Gamma_{\mathrm{L}}=\Gamma_{\mathrm{R}} \equiv \Gamma / 2$.

The linear conductance through the system can be found from the Meir-Wingreen formula,

$$
G_{\sigma}=\frac{e^{2}}{h} \frac{4 \Gamma_{L \sigma} \Gamma_{R \sigma}}{\Gamma_{L \sigma}+\Gamma_{R \sigma}} \int \mathrm{d} \omega \frac{\partial f(\omega)}{\partial \omega} \Im\left\langle\left\langle d_{1 \sigma} \mid d_{1 \sigma}^{\dagger}\right\rangle\right\rangle^{\mathrm{ret}},
$$

where $f(\omega)$ is the Fermi-Dirac distribution function and $\left\langle\left\langle d_{1 \sigma} \mid d_{1 \sigma}^{\dagger}\right\rangle\right\rangle^{\text {ret }}$ denotes the Fourier transform of the retarded Green function of the first dot, which we calculate with the aid of NRG $[9,10]$. In calculations we assume the following parameters (the first dot is noninteracting): $U_{2}=0.5 D, \Gamma=0.02 D, t=0.04 D$ and $p=0.4$, where $D \equiv 1$ is the band halfwidth used as energy unit. 


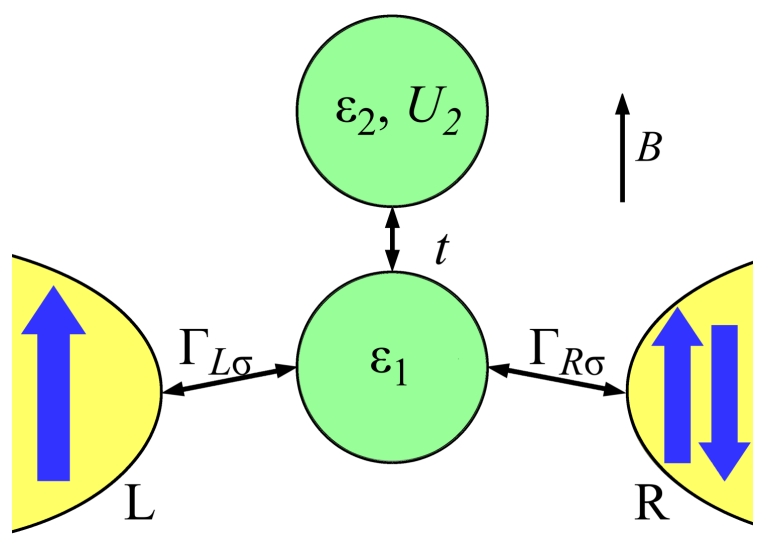

Fig. 1. Schematic of DQD in a T-shape geometry coupled to ferromagnetic leads with either parallel or antiparallel alignment of magnetizations. The first dot is coupled to the leads with strength $\Gamma_{\mathrm{L}(\mathrm{R}) \sigma}$ and to the second dot via hopping $t$.

\section{Results and discussion}

In the following, we analyze the behavior of the spinresolved conductance $G_{\sigma}$ and the spin polarization $\mathcal{P}$, defined as $\mathcal{P}=\left(G_{\uparrow}-G_{\downarrow}\right) /\left(G_{\uparrow}+G_{\downarrow}\right)$, in both the antiparallel (AP) and parallel (P) magnetic configurations of the system.

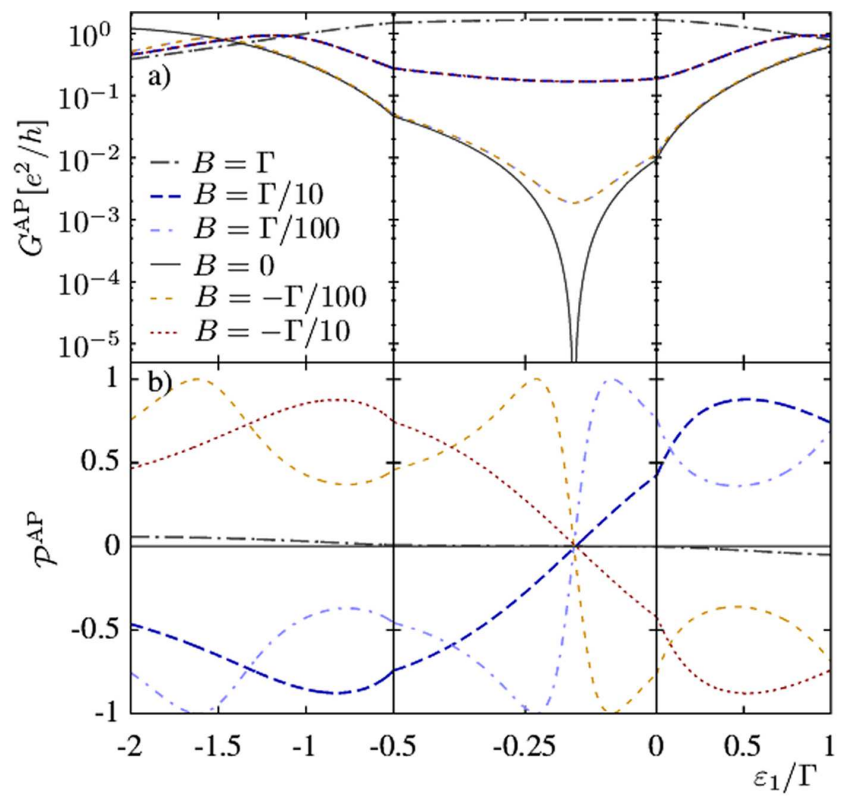

Fig. 2. Dependence of the linear conductance $G^{\mathrm{AP}}$ (a) and spin polarization $\mathcal{P}^{\mathrm{AP}}$ (b) on $\varepsilon_{1}$, for different strengths of magnetic field $B$ in the antiparallel configuration and for $\varepsilon_{2}=-U_{2} / 3$. The $x$-axis for $\varepsilon_{1} \in[-0.5,0]$ was multiplied by 3 to zoom in the range where the most interesting features occur (curves are smooth in homogeneous scale). In (a) curves for different signs of $B$ overlap.

In the antiparallel configuration, for left-right symmetric systems as considered here, the couplings for spin-up and spin-down DQD levels become equal and the system behaves as if coupled to nonmagnetic leads, except for additional factor of $\left(1-p^{2}\right)$ in $G_{\sigma}$. Figure 2 shows the linear conductance and spin polarization in the antiparallel configuration as a function of $\varepsilon_{1}$ for $\varepsilon_{2}=-U_{2} / 3$. As can be clearly seen in Fig. $2 \mathrm{a}$, for $B=0$, there is a strong antiresonance in $G^{\mathrm{AP}}$ due to the Fano destructive interference. It occurs for $\varepsilon_{1}=\varepsilon_{0} \approx-\Gamma / 6$. The fact that $\varepsilon_{0} \neq 0$ comes form the lack of electron-hole symmetry in the system, since $\varepsilon_{2}=-U_{2} / 3$. We note that for $\varepsilon_{2}=-U_{2} / 2$ the conductance in the presence of $B$ was analyzed in Ref. [6] for nonmagnetic leads.

While for $B=0$ the suppression of $G^{\mathrm{AP}}$ is complete, the presence of magnetic field weakens this effect and leads to finite conductance. This is due to the fact that finite $B$ removes spin degeneracy and the conditions for Fano destructive interference become different in each spin channel. Then, if $G_{\sigma}^{\mathrm{AP}} \rightarrow 0$ for given $\varepsilon_{1}, G_{\bar{\sigma}}^{\mathrm{AP}} \neq 0$, so that the total conductance, $G^{\mathrm{AP}}=G_{\uparrow}^{\mathrm{AP}}+G_{\downarrow}^{\mathrm{AP}}$, is finite in the whole range of $\varepsilon_{1}$, provided $B \neq 0$, see Fig. 2a. This holds even for very weak $B$, while for strong magnetic field a dip in $G^{\mathrm{AP}}$ changes into a wide peak, reaching $G^{\mathrm{AP}}=2\left(1-p^{2}\right) e^{2} / h$. Another feature visible in Fig. $2 \mathrm{a}$ is the approximate symmetry of the linear conductance around the point $\varepsilon_{1}=\varepsilon_{0}$.

$G^{\mathrm{AP}}$ does not depend on the sign of $B$, only on its magnitude, this is contrary to the spin polarization, which is shown in Fig. 2b. Since for $B=0$ nothing perturbs the spin $S U(2)$ symmetry, $\mathcal{P}^{\mathrm{AP}}=0$ in this case. However, even as tiny magnetic field as $B=\Gamma / 100$ is sufficient to cause $\mathcal{P}^{\mathrm{AP}}$ to reach \pm 1 . In agreement with intuition, the change of sign of $B$ leads to the change of sign of $\mathcal{P}^{\mathrm{AP}}$ and all the curves are approximately antisymmetric around $\varepsilon_{1}=\varepsilon_{0}$. Nevertheless, the dependence of $\mathcal{P}^{\mathrm{AP}}$ on $\varepsilon_{1}$ for $B= \pm \Gamma / 100$ is highly non-trivial. It displays two local maxima with $\mathcal{P}^{\mathrm{AP}} \approx 1$ and two minima with $\mathcal{P}^{\mathrm{AP}} \approx-1$. On the other hand, the dependence of $\mathcal{P}^{\mathrm{AP}}$ on $\varepsilon_{1}$ for stronger $B$ is less spectacular, i.e. the achieved values of $\mathcal{P}^{\mathrm{AP}}$ are now much smaller, $\left|\mathcal{P}^{\mathrm{AP}}\right|<0.1$, see the curves for $B= \pm \Gamma$ in Fig. $2 \mathrm{~b}$. This holds also outside the range of Fig. 2, for $-15 \Gamma<\varepsilon_{1}<15 \Gamma$.

Consider now the case of parallel magnetic configuration. Now the couplings for spin-up and spin-down are different leading to different level renormalization for each spin direction. This gives rise to a spin splitting of the levels even in the case of $B=0$ [7]. The magnitude and sign of this ferromagnetic-contact induced exchange field can be tuned by changing the level position. Moreover, it is clearly an interaction effect since it vanishes for vanishing Coulomb correlations and at the particle-hole symmetry point of the model. In the case studied here with noninteracting first dot, the exchange field develops in the second dot. The hybridization of the second dot is $\Gamma_{2 \sigma}=t^{2} / \Gamma_{\sigma}$, and, clearly, the dependence of $\Gamma_{2 \sigma}$ on $p$ is opposite to the that of $\Gamma_{\sigma}[7,8]$. Consequently, for given levels' position, the sign of exchange field is opposite as compared to single dot case. This is reflected in the $B$-dependence of $G$ and $\mathcal{P}$, as discussed in the sequel. 
The dependence of linear conductance $G^{\mathrm{P}}$ and spin polarization $\mathcal{P}^{\mathrm{P}}$ on $\varepsilon_{1}$ is shown in Fig. 3. One can see that even for $B=0$ the suppression of the conductance due to Fano destructive interference is only partial. Due to the presence of exchange field, Fano antiresonances in different spin channels are shifted against each other, leading to finite conductance $G^{\mathrm{P}}$ for all $\varepsilon_{1}$. Moreover, the spin polarization is very high, $\mathcal{P}^{\mathrm{P}} \approx 1$, except for the point where $G^{\mathrm{P}}$ is minimum, at which the spin polarization changes sign and becomes $\mathcal{P}^{\mathrm{P}} \approx-1$. Thus, without any external magnetic field, due to the presence of exchange field, one can obtain perfect spin polarization of the linear conductance, which can be tuned by changing the level position. This undoubtedly interesting result is analyzed in more detail in Ref. [11].

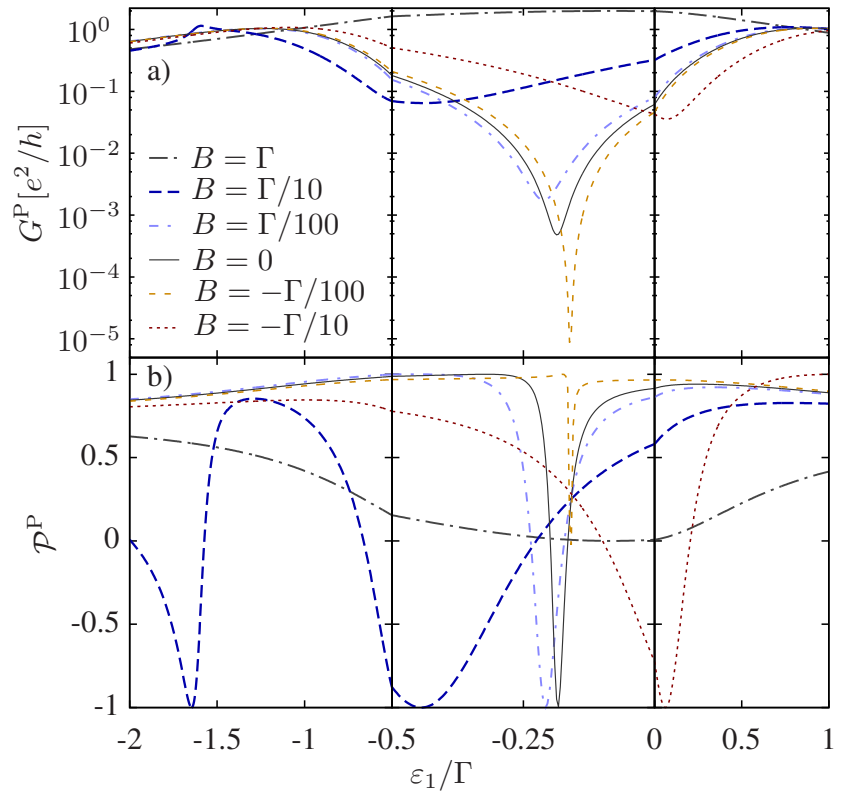

Fig. 3. The same as Fig. 2 calculated for the parallel magnetic configuration of the system.

In the presence of magnetic field, the operation of the device can be changed. If one turns on small negative $B$, see the curves for $B=-\Gamma / 100$ in Fig. 3, the Fano antiresonance becomes restored and $\mathcal{P}$ is almost constant showing only small dip without any sign change. This is due to the fact that this magnetic field compensates the splitting induced by the exchange field. Note that this is contrary to single dot case, where to compensate for exchange field one needs to apply $B$ along the magnetization of the leads $(B>0)[8]$. This is related with different dependence of the couplings on $p$ discussed above.

For small positive field, see the case of $B=\Gamma / 100$ in Fig. 3, the minimum of $G^{\mathrm{P}}$ is slightly shifted and a bit shallower, while the dip of $\mathcal{P}^{\mathrm{P}}$ reaches -1 and is wider as compared to the case of $B=0$. This can be understood by realizing that positive $B$ enhances the splitting induced by the exchange field. The splitting can be enhanced further with larger $B$ leading to even broader minimum in both $G^{\mathrm{P}}$ and $\mathcal{P}^{\mathrm{P}}$, see the case of $B=\Gamma / 10$ in Fig. 3. Interestingly, a second minimum of $\mathcal{P}^{\mathrm{P}}$ occurs at $\varepsilon_{1} \approx-1.65 \Gamma$. At this point $G^{\mathrm{P}}$ is enhanced and exhibits a small local maximum.

The change of sign of $B$ is in this case qualitatively equivalent to change of the curve to its mirror image with respect to the point where $G^{\mathrm{P}}$ has minimum. This is due to the fact that for $|B| \gtrsim \Gamma / 10$, the magnetic field surpasses the exchange field, thus decreasing its influence on transport. In fact, for magnetic fields of the order of the coupling strength, $|B|=\Gamma$, the spin polarization becomes much suppressed, it is clearly smaller than unity and does not change sign.

Finally, we note that large spin polarization is also present in the case when the first dot is interacting. Then, the exchange field plays an important role on both dots and it can lead to further enhancement of the range of parameters where perfect spin polarization occurs [11].

\section{Acknowledgments}

This work was supported by the Polish Ministry of Science and Higher Education through a research project No. N N202 199739 in years 2010-2013. I.W. also acknowledges support from the EU grant No. CIG-303 689.

\section{References}

[1] W.G. van der Wiel, S. De Franceschi, J.M. Elzerman, T. Fujisawa, S. Tarucha, L.P. Kouwenhoven, Rev. Mod. Phys. 75, 1 (2003).

[2] U. Fano, Phys. Rev. 124, 1866 (1961).

[3] A.C. Hewson, The Kondo Problem to Heavy Fermions, Cambridge University Press, Cambridge 1993.

[4] S. Sasaki, H. Tamura, T. Akazaki, T. Fujisawa, Phys. Rev. Lett. 103, 266806 (2009).

[5] R. Žitko, Phys. Rev. B 81, 115316 (2010).

[6] L.G.G.V. Dias da Silva, E. Vernek, K. Ingersent, N. Sandler, S.E. Ulloa, Phys. Rev. B 87, 205313 (2013).

[7] J. Martinek, Y. Utsumi, H. Imamura, J. Barnaś, S. Maekawa, J. König, G. Schön, Phys. Rev. Lett. 91, 127203 (2003).

[8] M. Gaass, A.K. Hüttel, K. Kang, I. Weymann, J. von Delft, Ch. Strunk, Phys. Rev. Lett. 107, 176808 (2011).

[9] K.G. Wilson, Rev. Mod. Phys. 47, 773 (1975).

[10] We use the open-access Budapest NRG code, O. Legeza, C.P. Moca, A.I. Tóth, I. Weymann, G. Zaránd, arXiv:0809.3143 (2008), unpublished. The code is available at: http://www.phy.bme.hu/dmnrg/.

[11] K.P. Wójcik, I. Weymann, Phys. Rev. B 90, 115308 (2014). 